\title{
Review
}

\section{The utility of transcription factors for manipulation of floral traits}

\author{
Masahito Shikata, ${ }^{1}$ Masaru Ohme-Takagi ${ }^{1,2, *}$ \\ ${ }^{1}$ Research Institute of Genome-based Biofactory, National Institute of Advanced Industrial Science and Technology, \\ Tsukuba, Ibaraki 305-8562, Japan; ${ }^{2}$ Core Research for Evolutional Science and Technology, Japan Science and \\ Technology Agency, Kawaguchi, Saitama 332-0012, Japan \\ *E-mail: m-takagi@aist.go.jpＴel: +81-29-861-2641 Fax: +81-29-861-3026
}

Received November 15, 2007; accepted December 20, 2007 (Edited by Y. Ozeki)

\begin{abstract}
Cross-pollination is an effective method of breeding flowering plants to produce novel variation. However, this strategy is often protracted or demands repeated crossing for several generations to obtain desired traits. Recently, genemodification technologies have been utilized in plant breeding for manipulation of floral traits. Many homeotic genes that regulate flower development have been shown to encode transcription factors. In this review we describe the utility of transcription factors and a novel gene-silencing technology, the CRES-T system, to effectively manipulate floral traits. When AGAMOUS $(A G)$ and APETALA3 (AP3) were subjected to this system in Arabidopsis, ag-like and ap3-like phenotypes, respectively, were induced with high efficiency as a dominant trait. Plant transcription factors are conserved between different species to some extent and consequently the chimeric repressor derived from Arabidopsis can be applied to other species without any modification. By applying this system, new floral color and shape phenotypes were obtained in Torenia fournieri and Ipomoea nil. Since the CRES-T system is able to overcome the problem of gene redundancy, polyploid plants may be also manipulated with this system. In addition, with the CRES-T system modification of the flower morphology of plants for which limited genome sequence information is available can be expected.
\end{abstract}

Key words: $\mathrm{ABC}$ model, CRES-T system, floral trait, transcription factor.

Flowers are a characteristic organ in angiosperm and consist of sepals, petals, stamens and carpels. Petals are the most conspicuous organ in flowers because of their variation in colors and shapes. Some plants such as Tulipa gesneriana have tepals instead of sepals and petals and Hydrangea have degenerated petals and outstanding sepals in their flowers. The breeding and commercial production of cut flowers and ornamental plants is of major economic importance. Therefore, production of novel variation in flowers is an important focus of plant breeding. Through crossing different genotypes and selection for particular traits among the hybrid progeny, large numbers of phenotypically divergent varieties have been developed from the wild, less genetically diverse species. For example, wild Eustoma grandiflorum from North America has only purple-blue flowers, but a great number of color variations of this species are now available. Crosspollination is a useful strategy for crop improvement and for production of genetically stable new varieties. However, this strategy may be protracted and require repeated crossing over several generations to acquire new and desired traits.

On the other hand, gene-modification technologies are a powerful tool to alter plant traits rapidly. Previously, mutagenesis by chemical or UV treatment was used to induce genetic variation. Recently, ion-beam irradiation has been shown to be a useful tool for mutagenesis (Shirley et al. 1992). However, due to the presence of redundant genes in the plant genome, this strategy is not always effective to obtain desired phenotypes especially in polyploid plants. Transgene expression has been shown to be an effective and more rapid means of introducing a desired phenotype into a target plant. This technology has been applied to plants to transfer agriculturally useful traits, such as insect or herbicide resistance (Comai and Shen 1983; Bates et al. 2005), as well as to manipulate floral traits. Alterations of petal color have been reported by ectopic expression or introducing antisense for genes related to pigmentation biosynthesis (Forkmann and Martens 2001). However, attempts to manipulate floral traits have not been fully achieved, probably due to limited availability of genomic information regarding specific floral traits in horticultural plants.

Plant gene expression is largely controlled at the transcription level and thus transcription factors play a pivotal role in gene regulation. Because transcription factors are capable of controlling the expression of multiple genes, some have been shown to act as a 
'master' regulator for various phenotypes. Many mutants with flower abnormalities have defective transcription factors (reviewed by Jack 2004). Therefore, it can be predicted that transcription factors are a potential tool for the easy and efficient manipulation of floral traits. Recently, we developed a novel gene-silencing system using the chimeric transcriptional repressor, which is designated chimeric repressor gene-silencing technology (CRES-T). This system can induce a phenotype that was not expressed by antisense or gene knockout lines (Hiratsu et al. 2003).

In this review, we highlight the various roles that transcription factors play in flower development and the utility of the CRES-T system for manipulation of floral traits.

\section{Many transcription factors regulate floral traits}

Mutant analyses of Arabidopsis thaliana and Antirrhinum majus have revealed that a number of genes that regulate flower development encode transcription factors. Some of them are categorized into $\mathrm{ABC}$ genes of the wellknown $\mathrm{ABC}$ model, which specify floral organ identities (Coen and Meyerowitz 1991). According to the ABC model, four floral organs (sepals, petals, stamens and carpels) are arranged in four concentric whorls from the outermost sepals to the innermost carpels. These organs are specified by a combination of three classes of genes, namely A, B and C (Figure 1A). Mutation in ABC genes causes homeotic conversion of floral organs. Most of $\mathrm{ABC}$ genes encode MADS-box transcription factors. One of the most striking phenotype is the mutation of the $A G$ gene in Arabidopsis. Stamens of the ag mutant are converted into petals, and the fourth whorl bears another $a g$ flower, resulting in an indeterminate flower with a repetitive pattern of sepals, petals and petals (Yanofsky et al. 1990). The flower of ag displays a similar appearance to that of a rose or double-flowered cherry blossom, which are believed to be a defect of a homologous gene of $A G$ (Kitahara et al. 2004). One of the excellent aspects of the $\mathrm{ABC}$ model is that it can be adapted to most flowering plants with some modification. Many nongrass monocot such as Tulipa gesneriana have petaloid organs, called tepals, in first and second whorls and it is interpreted by modified $\mathrm{ABC}$ model which $\mathrm{B}$ class gene expression expanded to first whorl (Figure 1B; reviewed by Kanno et al. 2007). Thus, modification of $A B C$ genes will make it possible to change floral traits. Besides $A G$, other ABC genes, namely APETALA1 (AP1), APETALA3 $(A P 3)$ and PISTILLATA (PI), also encode MADS-box family transcription factors (Mandel et al. 1992; Goto and Meyerowitz 1994; Jack et al. 1992; Yanofsky et al. 1990), while APETALA2 belongs to the AP2/ERF family (Jofuku et al. 1994).

A number of genes for transcription factors that do not belong to $\mathrm{ABC}$ genes also affect flower development.
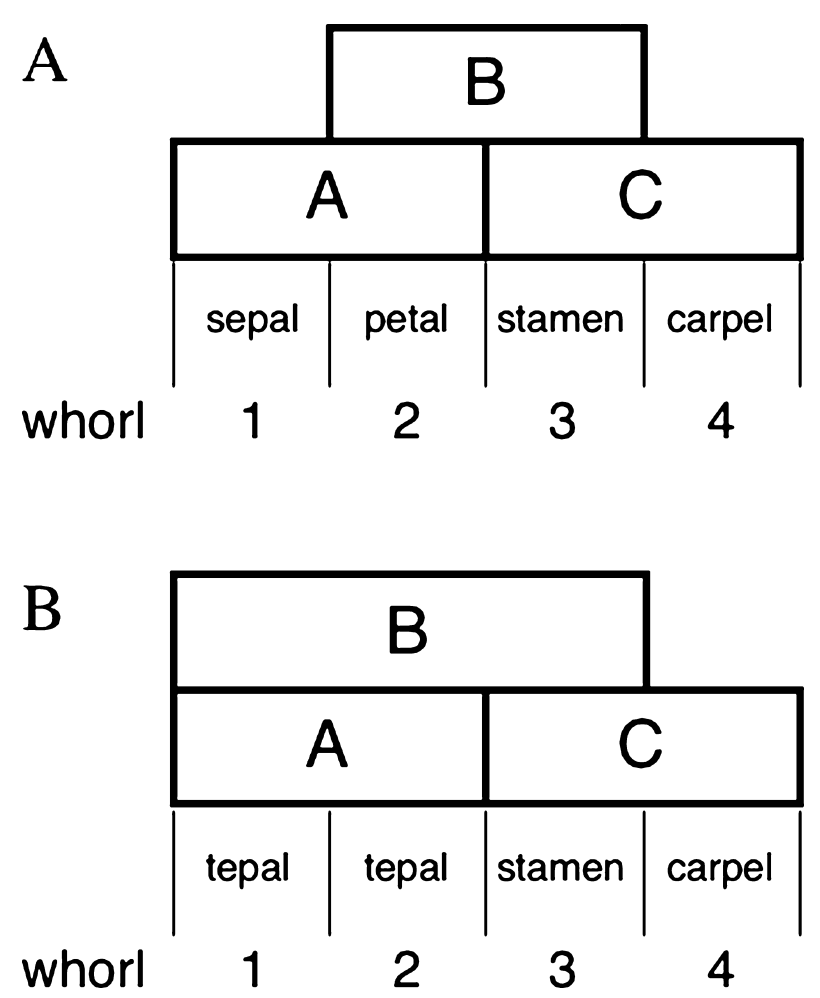

Figure 1. $\mathrm{ABC}$ model. (A) Classical $\mathrm{ABC}$ model. The combination of $\mathrm{A}, \mathrm{B}$ and $\mathrm{C}$ class genes specifies respective floral organs. (B) Modified ABC model. Expression of class B gene expands to the first whorl, resulting similar type of floral organs to those in second whorl.

The SUPERMAN (SUP) gene that encodes the zincfinger transcription factor negatively regulates expression of $A P 3$ and $P I$, and the sup mutation increases the number of stamens (Sakai et al. 1995). In contrast, mutation in the HANABA TARANU gene, which encodes the GATA-type zinc-finger protein, results in decreased number of floral organs (Zhao et al. 2004). PERIANTHIA $(P A N)$ encodes a bZIP protein and the pan mutant flowers have five sepals, five petals and five stamens whereas wild type flowers have four sepals, four petals and six stamens (Running and Meyerowitz 1996; Cuang et al. 1999). Wild type Antirrhinum has zygomorphic flowers, whereas mutation in both CYCLOIDEA and DICHOTOMA, which encode TCP family transcription factors, results in actinomorphic flowers (Luo et al. 1996; Cubas et al. 1999). These studies illustrate that, because a number of transcription factors play a role in the control of flower development, transcription factors are a useful tool for the manipulation of floral traits and generation of novel variation.

\section{Function of transcription factors is conserved between plant species}

Recently, information from genomic and expressed sequence tags has accumulated in various plants, especially in model plants including Arabidopsis thaliana, Oryza sativa, Zea mays, Medicago truncatula 
and Antirrhinum majus. This information revealed that plants have similar sets of transcription factors. ABC genes or their homologues are conserved among plants and the $\mathrm{ABC}$ model can be applied to most flowering plants. For example, Arabidopsis AP1, AP3, PISTILLATA and AG correspond to Antirrhinum SQUAMOSA, DEFICIENS, GLOBOSA and PLENA, respectively (Huijser et al. 1992; Schwarz-Sommer et al. 1992; Tröbner et al. 1992; Bradley et al. 1993). These MADSbox transcription factors control floral organ identities by forming particular complexes and the patterns of combination of MADS-box transcription factors are also conserved between Arabidopsis and Antirrinum (EgeaCortines et al. 1999; Honma and Goto 2001). In addition, formation of similar complexes was also reported in Petunia and Chrysanthemum (Ferrario et al. 2003; Shchennikova et al. 2004).

Conservation of the function of transcription factors between different species has been confirmed by complementation analysis between different species. If two homologous genes derived from different species have the same function, it is possible to complement the mutation of each other. For example, homologues of the Arabidopsis $L E A F Y$ ( $L F Y$ ) gene were found in various flowering plants and they could fully complement the lfy mutant (Maizel et al. 2005). These results indicate that transcription factors of Arabidopsis may be used for manipulation of floral traits in other flowering plants without any modification.

\section{Application of a gene-silencing system for manipulation of floral organ development}

Several transcription factors that identify floral organs have been shown to act as master regulators and are often conserved in many flowering plants. These suggest that modification of transcription factor that is expressed in flowers would be effective for the manipulation of flower traits. RNAi technology was applied to ABC genes to suppress $A G$ in Arabidopsis and SUPERWOMAN1 $(S P W 1)$ in rice (Chuang and Meyerowitz 2000; Xiao et al. 2003). However, it also revealed that a large number of plant genes are highly duplicated due to genome duplication or polyploidy (Moor and Purugganan 2005). In the presence of redundant genes, downregulation of a single gene, such as by gene knockout or RNAi, often fails to induce a visible phenotype (Figure 2A).

Recently, a novel gene-silencing system using a chimeric transcriptional repressor, designated CRES-T system, has been shown to be a useful tool to induce the defective phenotype. Moreover, the CRES-T system overcomes such difficulty of gene redundancy (Hiratsu et al. 2003; Matsui et al. 2005; Mitsuda et al. 2005; Mitsuda et al. 2007; Koyama et al. 2007). In this system, a chimeric repressor in which transcription factor fused to repression domain named SRDX, which derived from the ERF-associated amphiphilic repression (EAR) motif, suppresses expression of target genes (Figure 2B). As a result, the transgenic plant that expresses the chimeric repressor exhibits a phenotype similar to that of the lossof-function allele of the transcription factor gene (Hiratsu et al. 2003). Arabidopsis transgenic plants that express the chimeric $A G$ repressor (35S:AGSRDX) showed a similar phenotype to the ag mutant (Figure $2 \mathrm{C}$; Mitsuda et al. 2006; Yanofsky et al. 1990). Arabidopsis AP3, a B class gene, specifies petal and stamen identities; development of these organs fails in the ap3 mutant (Bowman et al. 1989; Jack et al. 1992). Transgenic plants that express the chimeric AP3 repressor (35S:AP3SRDX) mimicked the ap3 flower in Arabidopsis (Figure 2D; Mitsuda et al. 2006).

The advantage of the CRES-T system to other genesilencing technologies is that the chimeric repressor acts dominantly not only to endogenous transcription factors but also to functionally redundant factors, and that it can induce the defective phenotype as a dominant trait. We previously demonstrated that the CRES-T system could induce a defective phenotype even in the presence of redundant factors using the chimeric $C U C 1$ repressor as a model (Hiratsu et al. 2003). With regard to the phenotype in flowers, expression of the chimeric repressor for Arabidopsis TCP3, a member of the TCP family, induced wavy and serrated sepals and petals as well as leaf serration, although no visible phenotype was observed in their T-DNA inserted lines (Koyama et al. 2007). Functional redundancy is a major obstacle not only for functional analysis but also for manipulation of plant traits. Because the CRES-T system can induce a phenotype that was not expressed in antisense or knockout lines due to the presence of functionally redundant factors, this system is a powerful tool for manipulation of polyploid plants, such as hexaploid Chrysanthemum morifolium.

\section{Application of CRES-T system to various plants}

The CRES-T system functions also in plants other than Arabidopsis. Rice SPW1 is an ortholog of AP3 and in the spw1 mutant, lodicules and stamens were transformed to palea-like organs and carpels, respectively (Nagasawa et al. 2003). When the chimeric SPW1 repressor was expressed in rice, transgenic lines have $s p w 1$-like flowers (Mitsuda et al. 2006). In these transgenic plants, the rice SPW1 gene was used for construction of the chimeric repressor, while the sequence of the repression domain was identical to the sequence used in Arabidopsis. As described earlier, the function of transcription factors is usually conserved between plant species. This suggests that the chimeric repressor constructed with an Arabidopsis transcription factor can be applied to other plants without any modification or with minor modification such as alteration of the promoter or 

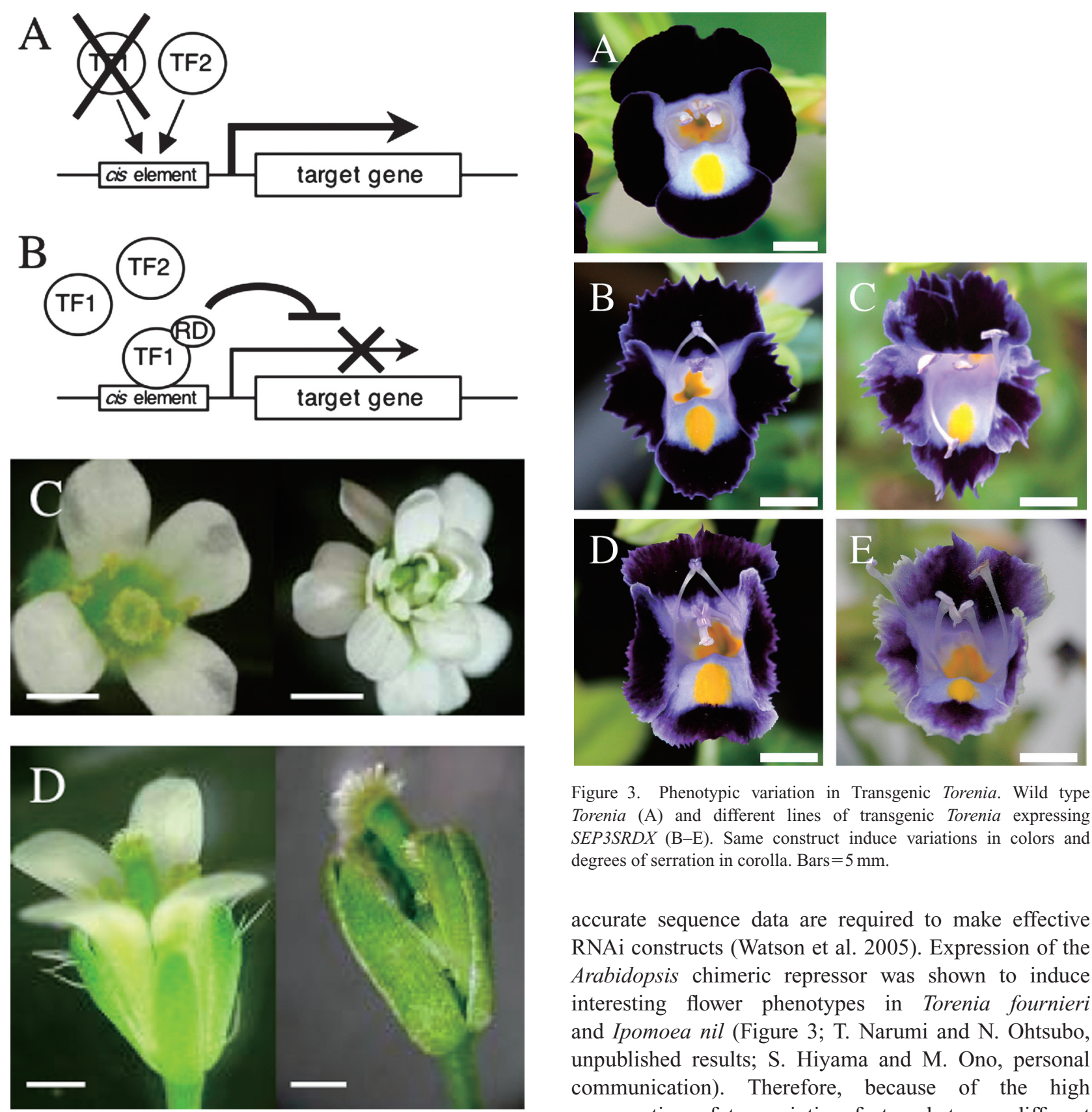

Figure 2. CRES-T system. (A) Existence of the functional redundancy of genes for plant transcription factors (TFs) often results in failure to show defective phenotype in single mutant (represented as $\mathrm{X}$ ). (B) A chimeric repressor in which transcription factor fused to repression domain (RD) act dominantly not only endogenous gene (TF1) but also functionally redundant gene (TF2), resulting in defective phenotypes. (C) Wild type flower (left) and 35S:AGSRDX flower (right). (D) Wild type flower (left) and 35S:AP3SRDX flower (right). Bars $=0.5 \mathrm{~mm}$.

selection marker. Although it would be better to use the gene of the host plant for maximal efficiency of the chimeric repressor, the genomic information necessary for construction of transgenes is not always available, especially in horticultural plants. For this reason RNAi technology cannot be applied to a plant for which limited sequence information is available because relatively

Figure 3. Phenotypic variation in Transgenic Torenia. Wild type Torenia (A) and different lines of transgenic Torenia expressing SEP3SRDX (B-E). Same construct induce variations in colors and degrees of serration in corolla. Bars $=5 \mathrm{~mm}$.

accurate sequence data are required to make effective RNAi constructs (Watson et al. 2005). Expression of the Arabidopsis chimeric repressor was shown to induce interesting flower phenotypes in Torenia fournieri and Ipomoea nil (Figure 3; T. Narumi and N. Ohtsubo, unpublished results; S. Hiyama and M. Ono, personal communication). Therefore, because of the high conservation of transcription factors between different species, it is possible to apply the CRES-T system to plants for which genomic information is unavailable.

\section{Advantage of transgenic plants for manipulation of floral traits}

Generally, gene mutation, in most cases null mutants, usually leads to stable and invariable phenotypes between generations or lines. On the other hand, phenotypes induced by a transgene are often varied mainly because of the different level of expression between lines. These variations are sometimes troublesome due to low phenotypic stability but at the same time such variation is useful to isolate a diversity of phenotypes. Such variation derived from a single construct can be used to obtain flowers with a new color or shape. For example, a 
chimeric repressor of SEP3, floral organ identity gene, has been shown to induce a range of floral colors and degrees of serration in corolla in transgenic Torenia (Figure 3; T. Narumi and N. Ohtsubo, unpublished results).

It is possible to manipulate and confer a new trait in a specific tissue by contriving the spatial and temporal expression of the transgene, such as that driven by a tissue-specific promoter. Moreover, alteration of the promoter sequence may induce another phenotype that will not be induced by the constitutive promoter, such as the CaMV35S promoter. In Arabidopsis, several chimeric repressor constructs are reported to induce a phenotype only when it was driven by its own promoter (Mitsuda et al. 2005; Ito et al. 2007).

Some plants with interesting floral phenotypes are sterile. It is difficult to maintain such sterile lines even if the floral phenotype is very rare and worth preserving. In contrast, the sterile phenotype induced by a transgene is reproducible.

\section{Conclusion}

Arabidopsis contains about 2,000 genes for transcription factors (Guo et al. 2005). More than 250 of these are highly expressed in the floral meristem and floral organs (Schmid et al. 2005). Chimeric repressors derived from the transcription factors that are expressed in floral organs may induce novel phenotypes that have not been expressed in screening with previous mutant lines, such as gene knockout lines, because the CRES-T system overcomes the problem of functional redundancy of transcription factors. It is predicted that it will be possible to create plant varieties with new floral traits by applying the CRES-T system to a diversity of flowering plants. In addition, comparison of the phenotype in diverse species in which the same chimeric repressor was transformed may reveal the function of the transcription factor and enhance our understanding of the evolution of flowers.

\section{Acknowledgements}

This work was supported by a Grant-in-Aid "Research Project for Utilizing Advanced Technologies in Agriculture, Forestry and Fisheries" from the Research Council, Ministry of Agriculture, Forestry and Fisheries of Japan, grant No.1782.

\section{References}

Bates SL, Zhao JZ, Roush RT, Shelton AM (2005) Insect resistance management in GM crops: past, present and future. Nat Biotechnol 23: 57-62

Bowman JL, Smyth DR, Meyerowitz EM (1989) Genes directing flower development in Arabidopsis. Plant Cell 1: 37-52

Bradley D, Carpenter R, Sommer H, Hartley N, Coen E (1993) Complementary floral homeotic phenotypes result from opposite orientations of a transposon at the plena locus of Antirrhinum. Cell 15: 85-95

Chuang CF, Meyerowitz EM (2000) Specific and Heritable genetic interference by double-stranded RNA in Arabidopsis thaliana. Proc Natl Acad Sci USA 97: 4985-4990

Chuang CF, Running MP, Williams RW, Meyerowitz EM (1999) The PERIANTHIA gene encodes a bZIP protein involved in the determination of floral organ number in Arabidopsis thaliana. Genes Dev 13: 334-344

Coen ES, Meyerowitz EM (1991) The war of the whorl: genetic interactions controlling flower development. Nature 353: 31-37

Comai L, Shen LC, Stalk DM (1983) An altered aroA gene product confers resistance to the herbicide glyphosate. Science 221: 370-371

Cubas P, Lauter N, Doebley J, Coen E (1999) The TCP domain: a motif found in proteins regulating plant growth and development. Plant J 18:215-222

Egea-Cortines M, Saedler H, Sommer H (1999) Ternary complex formation between the MADS-box proteins SQUAMOSA, DEFICIENS and GLOBOSA is involved in the control of floral architecture in Antirrhinum majus. EMBO J 18: 5370-5379

Ferrario S, Immink RGH, Shchennikova A, Busscher-Lange J, Angenent GC (2003) The MADS box gene $F B P 2$ is required for SEPALLATA function in petunia. Plant Cell 15: 914-925

Forkmann G, Martens S (2001) Metabolic engineering and applications of flavonoids. Curr Opin Biotechnol 12: 155-160

Goto K, Meyerowitz EM (1994) Function and regulation of the Arabidopsis floral homeotic gene PISTILLATA. Genes Dev 8: $1548-1560$

Guo A, He K, Liu D, Bai S, Gu X, Wei L, Luo J (2005) DATF: a database of Arabidopsis transcription factors. Bioinformatics 10: $2568-2569$

Hiratsu K, Matsui K, Koyama T, Ohme-Takagi M (2003) Dominant repression of target genes by chimeric repressors that include the EAR motif, a repression domain, in Arabidopsis. Plant $J$ 34: 733-739

Honma T, Goto K (2001) Complexes of MADS-box proteins are sufficient to convert leaves into floral organs. Nature 409: 525-529

Huijser P, Klein J, Lönnig WE, Meijer H, Saedler H, Sommer H (1992) Bracteomania, an inflorescence anomaly, is caused by the loss of function of the MADS-box gene squamosa in Antirrhinum majus. EMBO J 11: 1239-1249

Ito T, Nagata N, Yoshiba Y, Ohme-Takagi M, Ma H, Shinozaki K (2007) Arabidopsis MALE STERILITY1 encodes a PHD-type transcription factor and regulates pollen and tapetum development. Plant Cell 19: 3549-3562

Jack T, Brockman LL, Meyerowitz EM (1992) The homeotic gene APETALA3 of Arabidopsis thaliana encodes a MADS box and is expressed in petals and stamens. Cell 68: 683-697

Jack T (2004) Molecular and genetic mechanisms of floral control. Plant Cell 16: S1-S17

Jofuku KD, den Boer BGW, Montagu MV, Okamuro JK (1994) Control of Arabidopsis flower and seed development by the homeotic gene APETALA2. Plant Cell 6: 1211-1225

Kanno A, Nakada M, Akita Y, Hirai M (2007) Class B gene expression and the modified $\mathrm{ABC}$ model in nongrass monocots. TSW Dev Embryol 2: 17-28

Kitahara K, Hibino Y, Aida R, Matsumoto S (2004) Ectopic expression of the rose AGAMOUS-like MADS-box genes 'MASAKO C1 and DI' causes similar homeotic transformation of sepal and petal in Arabidopsis and sepal in Torenia. Plant 
Sci 166: $1245-1252$

Koyama T, Furutani M, Tasaka M, Ohme-Takagi M (2007) TCP transcription factors control the morphology of shoot lateral organs via negative regulation of the expression of boundaryspecific genes in Arabidopsis. Plant Cell 19: 473-484

Luo D, Carpenter R, Vincent C, Copsey L, Coen E (1996) Origin of floral asymmetry in Antirrhinum. Nature 383: 794-799

Maizel A, Busch MA, Tanahashi T, Perkovic J, Kato M, Hasebe M, Weigel D (2005) The floral regulator LEAFY evolves by substitutions in the DNA binding domain. Science 308 : 260-263

Mandel MA, Gustafson-Brown C, Savidge B, Yanofsky MF (1992) Molecular characterization of the Arabidopsis floral homeotic gene APETALA1. Nature 360: 273-277

Matsui K, Hiratsu K, Koyama T, Tanaka H, Ohme-Takagi M (2005) A chimeric AtMYB23 repressor induces hairy roots, elongation of leaves and stems, and inhibition of the deposition of mucilage on seed coats in Arabidopsis. Plant Cell Phisiol 46: 147-155

Mitsuda N, Seki M, Shinozaki K, Ohme-Takagi M (2005) The NAC transcription factors NST1 and NST2 of Arabidopsis regulate secondary wall thickenings and are required for anther dehiscince. Plant Cell 17: 2993-3006

Mitsuda N, Hiratsu K, Todaka D, Nakashima K, YamaguchiShinozaki K, Ohme-Takagi M (2006) Efficient production of male and female sterile plants by expression of a chimeric repressor in Arabidopsis and rice. Plant Biotechnol $J$ 4: 352-332

Mitsuda N, Iwase A, Yamamomto H, Yoshida M, Seki M, Shinozaki K, Ohme-Takagi M (2007) NAC transcription factors, NST1 and NST3, are key regulators of the formation of secondary walls in woody tissue of Arabidopsis. Plant Cell 19: 270-280

Moore R, Purugganan MD (2005) The evolutionary dynamics of plant duplicate genes. Curr Opin Plant Biol 8: 122-128

Nagawawa N, Miyoshi M, Sano Y, Satoh H, Hirano H, Sakai H, Nagato Y (2003) SUPERWOMAN1 and DROOPING LEAF genes control floral organ identity in rice. Development 130 : 705-718

Running MP, Meyerowitz EM (1996) Mutation in the
PERIANTHIA gene of Arabidopsis specifically alter floral organ number and initiation pattern. Development 122: 1261-1269

Sakai H, Medrano LJ, Meyerowitz EM (1995) Role of SUPERMAN in maintaining Arabidopsis floral whorl boundaries. Nature 378: 199-203

Schmid M, Davison TS, Henz SR, Pape UJ, Demar M, Vingron M, Schölkopf B, Weigel D, Lohmann JU (2005) A gene expression map of Arabidopsis thaliana development. Nat Genet 37: 501-506

Schwarz-Sommer Z, Hue I, Huijser P, Flor PJ, Hansen R, Tetens F, Lönnig WE, Saedler H, Sommer H (1992) Characterization of the Antirrhinum floral homeotic MADS-box gene deficiens: evidence for DNA binding and autoregulation of its persistent expression thoughout flower development. $E M B O J$ 11: 251-263

Shchennikova AV, Shulga OA, Immink R, Skryabin KG, Angenent GC (2004) Identification and characterization of four chrysanthemum MADS-box genes, belonging to the APETALA1/FRUITFULL and SEPALLATA3 subfamilies. Plant Physiol 134: 1632-1641

Shirley BW, Hanley S, Goodman HM (1992) Effects of ionizing radiation of a plant genome: analysis of two Arabidopsis transparent testa mutations. Plant Cell 4: 333-347

Tröbner W, Ramirez L, Motte P, Hue I, Huijser P, Lönnig WE, Saedler H, Sommer H, Schwarz-Sommer Z (1992) GLOBOSA: a homeotic gene which interacts with DEFICIENS in the control of Antirrhinum floral organogenesis. EMBO J 11: 4693-4704

Watson JM, Fusaro AF, Wang MB Waterhouse PM (2005) RNA silencing platforms in plants. FEBS let 579: 5982-5987

Xiao H, Wang Y, Liu D, Wang W, Li X, Zhao X, Xu J, Zhai W, Zhu L (2003) Functional analysis of the rice AP3 homologue OsMADS16 by RNA interference. Plant Mol Biol 52: 957-966 Yanofsky MF, Ma H, Bowman JL, Drews GN, Feldmann KA, Meyerowitz EM (1990) The protein encoded by the Arabidopsis homeotic gene agamous resembles transcription factors. Nature 346: 35-39

Zhao Y, Medrano L, Ohashi K, Fletcher JC, Yu H, Sakai H, Meyerowitz EM (2004) HANABA TARANU is a GATA transcription factor that regulates shoot apical meristem and flower development in Arabidopsis. Plant Cell 16: 2586-2600 